\title{
Commutator errors in large-eddy simulation
}

\author{
Bernard J Geurts ${ }^{1,2}$ and Darryl D Holm ${ }^{3,4}$ \\ ${ }^{1}$ Department of Applied Mathematics, Multiscale Modeling and Simulation, NACM, \\ University of Twente, PO Box 217, 7500 AE Enschede, The Netherlands \\ 2 Anisotropic Turbulence, Fluid Dynamics Laboratory, Eindhoven University of Technology, \\ PO Box 513, 5300 MB Eindhoven, The Netherlands \\ ${ }^{3}$ Mathematics Department, Imperial College London, SW7 2AZ, London, UK \\ ${ }^{4}$ Computational and Computer Science Division, Los Alamos National Laboratory, Los Alamos, \\ NM 87 545, USA \\ E-mail: b.j.geurts@utwente.nl
}

Received 3 September 2005, in final form 22 December 2005

Published 15 February 2006

Online at stacks.iop.org/JPhysA/39/2213

\begin{abstract}
Commutator errors arise in large-eddy simulation of incompressible turbulent flow from the application of non-uniform filters to the continuity - and NavierStokes equations. For non-uniform, high order filters with bounded moments the magnitude of the commutator errors is shown to be of the same order as that of the turbulent stress fluxes. Consequently, one cannot reduce the size of the commutator errors independently of the turbulent stress terms by any judicious construction of such filter operators. Independent control over the commutator errors compared to the turbulent stress fluxes can, instead, be obtained by appropriately restricting the spatial variations of the filter-width and filter-skewness. For situations in which the dynamical consequences of the commutator errors are significant, e.g., near solid boundaries, explicit similarity modelling for the commutator errors is proposed, including the application of Leray regularization. The performance of this commutator error parametrization is illustrated for the one-dimensional Burgers equation. The Leray approach is found to capture the filtered flow with higher accuracy than conventional similarity modelling, which is particularly relevant for large filter-width variations.
\end{abstract}

PACS numbers: 47.27.Eq, 02.60.Cb

\section{Introduction}

In large-eddy simulation (LES) of turbulence one aims to predict the primary features of an unsteady flow without explicitly resolving all dynamically relevant length-scales [1]. The modelling of turbulent flow in large-eddy simulation starts from the introduction of a spatial, 
low-pass filter with externally specified filter-width $\Delta$. This allows one to locally distinguish flow-features with a length-scale larger than $\Delta$ from flow-features with length-scale smaller than $\Delta$. In the large-eddy simulation context, the former are referred to as 'resolved' while the latter class of flow-structures is identified as 'subgrid' or 'sub-filter'. During a simulation, the time-dependent resolved scales are explicitly calculated while the dynamic effects of the subgrid scales on the evolution of the resolved scales are represented through the introduction of an explicit 'subgrid' model. In the filtering approach to large-eddy simulation the spatial filter is traditionally assumed to be characterized by a single, spatially uniform operator, in particular, with a single value for $\Delta$ throughout the flow domain [2].

The desire to extend large-eddy simulation to flows in complex domains generally implies that one is confronted with strongly varying turbulence intensities within the flow-domain. In certain regions a nearly laminar flow may exist while a lively, fine-scale turbulent flow can be present simultaneously in other regions. As an example, one may think of flow over a backward facing step [3, 4] which displays very small turbulent boundary layer scales characteristic of a separated shear layer, while at other locations in the domain an unsteady but large-scale flow may be observed. Various other examples from nature and technology come to mind, which emphasize the need to incorporate heterogeneous smaller scales into the large-eddy approach in order to consistently address turbulent flows in complex situations.

In the filtering approach to large-eddy simulation, strongly non-uniform turbulence can be accommodated efficiently using a filter operator with non-uniform filter-width [5, 6]. The use of such filters, however, complicates the subgrid closure problem through the appearance of additional commutator errors [7]. These terms arise because non-uniform filtering does not commute with spatial differentiation. That is, $\overline{\partial_{x} u} \neq \partial_{x} \bar{u}$ where $\partial_{x} u$ denotes differentiation of the solution $u$ with respect to $x$ and the overline indicates the filter operation. In this paper we (i) establish the magnitude of the additional commutator error closure terms relative to the fluxes arising from the turbulent stress tensor, (ii) sketch under what conditions for the filter, the commutator errors are likely to be dynamically relevant and (iii) for such situations, propose and illustrate explicit similarity modelling and Leray regularization [8,9]. These findings encourage the further extension of the large-eddy approach to turbulence under realistic flow conditions and in complex flow domains.

The general subject-matter of commutator-errors in large-eddy simulation was also the topic of recent publications in $[11,12]$. Compared to these references, the current paper completes and complements in the three directions (i), (ii) and (iii) mentioned above. We describe similarities and differences with these earlier publications next.

In [11] the basic equations associated with non-uniform spatial filtering were collected and an a priori numerical assessment was given of the magnitude and spatial distribution of commutator-errors, based on DNS data of a turbulent mixing layer. Moreover, explicit similarity and gradient models for the commutator-error were compared with the actual commutator-errors. Emphasis was put on determining the correlation between model and actual commutator-error and on establishing the magnitude of the flux and the contribution to the overall dissipation. Several higher order numerical filters were included, both symmetric and skewed. In [12] a new explicit model for the commutator-error was proposed, based on the approximate Lagrangian interpretation of the dynamic effect of the commutator-error. An a priori analysis was given of the quality of the Lagrangian commutator-error model to approximate the contributions to the resolved kinetic energy dynamics and to represent the commutator-errors in the momentum equation.

The current paper is complementary to $[11,12]$ in three ways. First, a detailed theoretical analysis is given of the magnitude of commutator-errors and of the magnitude of the turbulent stress flux, for higher order filters with general spatial dependence of the filter-width and 
skewness distributions. This extends the treatment given in [11] which was restricted to constant skewness. Second, the possibilities offered by the Leray regularization approach [8,9] to derive explicit models for the commutator-error are worked out in full detail. Third, rather than restricting to a numerical a priori analysis, as was done in [11, 12], the dynamic consequences of the Leray model in actual simulations were studied. For this purpose the nonuniformly filtered Burgers equation was investigated. Predictions obtained with the new Leray commutator-error model were compared with filtered DNS data and with predictions based on an extended Bardina similarity model [13]. In summary, the current paper is dedicated to the theoretical analysis of general non-uniform filters, to the Leray regularization and to the dynamic consequences in actual numerical simulations of the Burgers equation.

The dynamic impact of the commutator errors can be clarified quite readily. As mentioned, the distinction between which flow-features are 'subgrid' and which are 'resolved', depends on the local filter-width $\Delta$. Therefore, spatial - and temporal variations in $\Delta$ imply additional energy-transfer and apparent interaction mechanisms among the scales in the computational model, besides the well-known physical energy cascade in the Navier-Stokes equations [10]. In fact, if a flow structure propagates, or is swept from a region of small filter-width into a region with strongly increased filter-width, it would appear as if (part of) this structure had turned from a 'resolved' to a 'subgrid' feature, merely by translation. The reverse can also be imagined, leading to the apparent emergence of resolved structures from a collection of subgrid scales. This Lagrangian interpretation of the dynamics of commutator errors suggests additional sources of local energy drain or backscatter, depending on the specific local filter-width variations in the direction of the instantaneous local flow [11, 12]. If the variations in the properties of the filter are sufficiently localized, an explicit parametrization of the corresponding closure terms appears to be required in order to maintain an efficient and accurate large-eddy representation of turbulent flow.

Progress toward successful large-eddy simulations of flows in and around complex geometries would appear to require that the mathematical and numerical formulations of the methods should at least remain compatible with basic aspects of the fluid dynamics equations. In particular, such developments may include discretizations on non-uniform, time-dependent, grids in complex geometry with corresponding non-uniform filter-width distributions in the flow domain. Significant developments related to the application of such non-uniform filters have appeared in the recent literature, e.g., $[14,15]$. These developments are primarily associated with the construction of specific high order filters. For such general ' $N$ th order' filters the magnitude of the commutator errors can be controlled to some degree. Specifically, recent papers claim that the size of the commutator errors can be sufficiently reduced for these terms to become negligible, simply by appropriately increasing the order of the adopted filter. This claim seems over-simplified, however, and its further investigation motivates the present study.

In the present paper we take a different approach to commutator errors. In fact, our main result shows that non-uniform filtering in general leads to commutator errors which are of the same formal order of magnitude as the turbulent stress fluxes in the filtered Navier-Stokes equations. This holds for any higher order filter and emphasizes the point that the magnitude of the commutator errors cannot be reduced independently of the turbulent stress fluxes by high order filtering alone; commutator errors cannot simply be 'filtered away' independently. Consequently, the question of determining the conditions under which commutator errors are dynamically relevant, is still largely open.

A recent numerical a priori study [11], involving turbulent flow in a mixing layer, showed that localized filter-width variations can lead to commutator errors about $25-30 \%$ of the size of the turbulent stress fluxes. Conversely, commutator errors were found quite negligible if the 
filter varies sufficiently smoothly, compared to dominant length-scales of the flow. This paper complements the previous numerical study from a more theoretical point of view. Rather than controlling the size of the commutator errors by increasing the order of the filter, the magnitude of these contributions is found to be influenced by controlling the spatial variations in the filter properties. We specify under what conditions the commutator errors can be expected to be negligible relative to the turbulent stress fluxes. That is, we identify situations such that the commutator errors do not require explicit closure. Alternatively, if these conditions cannot be satisfied, e.g., in a turbulent boundary layer [6], the introduction of an additional, explicit subgrid model for the commutator errors is summoned. For this purpose, we present an alternative formulation for large-eddy simulation based on Leray regularization $[8,9]$ which allows the systematic development of a subgrid model for the commutator errors. The results of explicit Leray modelling are compared with extended similarity subgrid modelling [13].

To provide a first illustration of explicit dynamical consequences of commutator errors and their similarity models, the evolution of running 'ramp-cliff' waves in the viscous Burgers equation is studied in one dimension. It is shown that the 'Leray-regularized' formulation provides a better representation of the non-uniformly filtered velocity field than the extended Bardina similarity model for this situation. In particular, the Leray approach simultaneously captures turbulent stress fluxes as well as commutator errors without increasing computational costs compared to the traditional case of uniform filters. The extension toward non-uniformly filtered turbulent flow in three dimensions is the subject of ongoing research.

The organization of the paper is as follows. In section 2 we consider how commutator errors arise in applying non-uniform spatial filters to the Navier-Stokes equations. Then, in section 3 the magnitude of the commutator errors is determined for general $N$ th order filters and compared with the turbulent stress fluxes. For cases in which the variations in the properties of the filter are sufficiently abrupt, explicit modelling of the dynamic effects of the commutator errors is required. Similarity and Leray models are introduced in section 4 and their performance for the Burgers equation is illustrated numerically. Finally, concluding remarks are collected in section 5.

\section{Commutator errors in the filtering approach to large-eddy simulation}

This section introduces a general class of non-uniform filters with compact support and applies them to the equations governing incompressible flow. The application of a non-uniform filter generates turbulent stress fluxes as well as commutator errors. Both groups of closure terms will be written as the commutator bracket of the filter operator and either the product operator, or the derivative operator. Consequently, the basic subgrid modelling problem in large-eddy simulation shares a few formal algebraic properties with the Poisson-bracket in classical mechanics $[16,17]$. The filtered velocity field is shown to acquire a nonzero divergence as a consequence of spatial variations in the filter properties. Finally, the effects of the commutator errors are described in terms of their contributions to the kinetic energy evolution. This description expresses the additional energy-transfer and interaction mechanisms associated with non-uniform filtering.

The usual formulation of the filtering approach to large-eddy simulation is based on convolution filters. This formulation assumes that the width of the filter is constant. However, the efficient extension of large-eddy simulation to turbulent flows in complex geometries or to cases with strong spatial variation of turbulence intensities, calls for the introduction of non-uniform filter-widths. For example, turbulent boundary layers or multi-phase flows and strongly localized combustion phenomena comprise three examples of turbulent flows whose efficient modelling naturally summons spatially varying filter-widths. 
The approach adopted here is based on a general compact-support filter, whose application in one spatial dimension is denoted by $\ell$ :

$$
\bar{u}(x, t)=\ell(u)(x, t)=\int_{x-\Delta_{-}(x, t)}^{x+\Delta_{+}(x, t)} \frac{H(x, \xi, t)}{\Delta(x, t)} u(\xi, t) \mathrm{d} \xi
$$

where $H(x, \xi, t)$ is the 'characteristic' filter function and $\Delta_{ \pm} \geqslant 0$ denote the upper - and lower bounding functions which define the filter-width $\Delta=\Delta_{+}+\Delta_{-}$. The filter $\ell$ is assumed to be normalized, i.e., $\ell(1)=1$. This class of filters can readily be extended to productfilters in three spatial dimensions by defining the composition $L=\ell_{1} \circ \ell_{2} \circ \ell_{3}$ where $\ell_{j}$ with $j=1,2,3$, represents filtering in the $x_{j}$-direction only, as in (1). In complex flows, spatial and temporal variations in turbulence-intensity pose different requirements on the local length-scale with which the flow should be represented in order to maintain an acceptable level of accuracy. Such situations may be addressed by allowing a non-uniform filter-width, as suggested in (1). However, the application of such filters gives rise to a number of additional closure terms, to which we turn next.

As is well known, incompressible flow is governed by the principles of conservation of mass and momentum. These can be expressed in terms of the continuity equation and Navier-Stokes equations as

$$
\partial_{j} u_{j}=0 \quad \partial_{t} u_{i}+\partial_{j}\left(u_{j} u_{i}\right)+\partial_{i} p-\frac{1}{R e} \partial_{j j} u_{i}=0, \quad i=1,2,3
$$

where $u_{j}$ is the component of the velocity $\mathbf{u}$ in the $x_{j}$-direction, $t$ denotes time and $\partial_{t}, \partial_{j}$ are the partial derivative operators with respect to $t$ and $x_{j}$ respectively. Moreover, $p$ is the pressure and $R e=\left(u_{r} \lambda_{r}\right) / v_{r}$ denotes the Reynolds number in terms of reference velocity $u_{r}$, length-scale $\lambda_{r}$ and kinematic viscosity $v_{r}$ [18]. Throughout, the summation convention is adopted, implying summation over repeated indices.

If one applies the filter $L$ to the incompressible flow equations, commutator errors may arise, e.g., if $\overline{\partial_{x} f}-\partial_{x} \bar{f}=L\left(\partial_{x} f\right)-\partial_{x}(L(f))=\left[L, \partial_{x}\right](f) \neq 0$. Here, the commutator error is written in terms of the commutator bracket $\left[L, \partial_{x}\right]$ of $L$ and the derivative operator $\partial_{x}$. One may show that $\left[L, \partial_{j}\right](f)=0$ for $j=1,2,3$, if and only if the filter $L$ is a convolution filter, which, by definition is spatially uniform. As for the continuity equation, we may formally write

$$
\partial_{j} \bar{u}_{j}=-\left[L, \partial_{j}\right]\left(u_{j}\right) .
$$

Hence the divergence of the non-uniformly filtered velocity differs from zero, i.e., $\bar{u}_{j}$ is not solenoidal, and the corresponding continuity equation is no longer in local conservation form. The term on the right-hand side corresponds to apparent local creation and annihilation of 'resolved' mass as a consequence of variations in $\Delta_{ \pm}$and $H$. Further developments and explicit parametrization are needed before this effect of non-uniform filtering can be consistently integrated into the large-eddy formulation. As an example, the form of (3) may motivate similarity modelling of the right-hand side: $\left[L, \partial_{j}\right]\left(u_{j}\right) \rightarrow\left[L, \partial_{j}\right]\left(\bar{u}_{j}\right)$. This yields specific contributions to the Poisson equation for the pressure and is a subject of current research.

Likewise, filtering the Navier-Stokes equations yields the following system of equations:

$$
\begin{gathered}
\partial_{t} \bar{u}_{i}+\partial_{j}\left(\bar{u}_{j} \bar{u}_{i}\right)+\partial_{i} \bar{p}-\frac{1}{R e} \partial_{j j} \bar{u}_{i}=-\left\{\left[L, \partial_{t}\right]\left(u_{i}\right)+\partial_{j}\left([L, S]\left(u_{i}, u_{j}\right)\right)+\left[L, \partial_{j}\right]\left(S\left(u_{i}, u_{j}\right)\right)\right. \\
\left.+\left[L, \partial_{i}\right](p)-\frac{1}{\operatorname{Re}}\left[L, \partial_{j j}\right]\left(u_{i}\right)\right\} .
\end{gathered}
$$

We observe that commutator brackets emerge involving the filter $L$ and the product operator $S(f, g)=f g$, as well as commutator brackets of $L$ and first- or second-order partial 
differentiation. Filtering a linear term such as $\partial_{t} u_{i}$ gives rise to a 'mean-flow' term $\partial_{t} \bar{u}_{i}$ and a corresponding commutator error $\left[L, \partial_{t}\right]\left(u_{i}\right)$. Filtering the nonlinear convective terms leads to two different types of closure terms. First, as in the case of uniform filtering, the divergence of the turbulent stress tensor $\tau_{i j}=\overline{u_{i} u_{j}}-\bar{u}_{i} \bar{u}_{j}=[L, S]\left(u_{i}, u_{j}\right)$ arises. The divergence of $\tau_{i j}$ will be referred to as the turbulent stress flux. Second, an associated commutator error $\left[L, \partial_{j}\right]\left(S\left(u_{i}, u_{j}\right)\right)$ emerges from filtering the convective fluxes. The local conservation form of the Navier-Stokes equations is no longer maintained as a result of the non-uniform filtering, similar to what was observed in (3) for the continuity equation.

Spatial filtering of the incompressible flow equations gives rise to an 'LES-template' [1] in which the 'Navier-Stokes' operator on the left-hand side of (4) acts on the filtered solution $\left\{\bar{u}_{i}, \bar{p}\right\}$. In addition, several unclosed terms arise. Of these, only the parametrization of the turbulent stress fluxes $\partial_{j}\left([L, S]\left(u_{i}, u_{j}\right)\right)=\partial_{j} \tau_{i j}$ has attracted much attention in the literature. However, the subgrid modelling problem associated with non-convolution filters entails various additional commutator errors. These terms require explicit modelling in case the spatial and temporal properties of the filter are sufficiently variable, as will be discussed in the next section.

The effects of the commutator errors can be effectively quantified by considering the turbulent kinetic energy equation. Multiplying (4) by $\bar{u}_{i}$ and summing over $i$ yields after some rewriting

$$
\begin{aligned}
\partial_{t}(k)+\partial_{j}\left(\bar{u}_{j} k\right) & =\frac{1}{R e} \bar{u}_{i} \partial_{j j} \bar{u}_{i}-\bar{u}_{i} \partial_{j} \tau_{i j}-k \partial_{j} \bar{u}_{j}-\bar{u}_{i} \partial_{i} \bar{p} \\
& -\bar{u}_{i}\left[L, \partial_{t}\right]\left(u_{i}\right)-\bar{u}_{i}\left[L, \partial_{j}\right]\left(u_{i} u_{j}\right)-\bar{u}_{i}\left[L, \partial_{i}\right](p)+\frac{1}{\operatorname{Re}} \bar{u}_{i}\left[L, \partial_{j j}\right]\left(u_{i}\right)
\end{aligned}
$$

where $k=\bar{u}_{i} \bar{u}_{i} / 2$ and we have used the identity $\partial_{j}\left(\bar{u}_{j} \bar{u}_{i} \bar{u}_{i}\right)=2 \bar{u}_{i} \partial_{j}\left(\bar{u}_{j} \bar{u}_{i}\right)-\bar{u}_{i} \bar{u}_{i} \partial_{j} \bar{u}_{j}$. On the right-hand side of (5) one identifies contributions due to the viscous terms and the turbulent stresses. Moreover, since $\partial_{j} \bar{u}_{j} \neq 0$, a specific commutator error contribution arises from the continuity equation (3) in addition to a pressure related term. The last four terms on the right-hand side of (5) represent effects of commutator errors in the momentum equations (4).

The resolved kinetic energy in a flow domain $\Omega$ of size $|\Omega|$ is defined as

$$
E=\frac{1}{|\Omega|} \int_{\Omega} \mathrm{d} \mathbf{x} \frac{1}{2} \bar{u}_{i} \bar{u}_{i}
$$

In a flow domain with periodic boundary conditions, the evolution of $E$ can be written in terms of the commutator error contributions as

$$
\begin{aligned}
|\Omega| \frac{\mathrm{d} E}{\mathrm{~d} t}=-\int_{\Omega} \mathrm{d} \mathbf{x}\left(\frac{1}{R e} \partial_{j} \bar{u}_{i} \partial_{j} \bar{u}_{i}-\tau_{i j} \partial_{j} \bar{u}_{i}\right)-\int_{\Omega} \mathrm{d} \mathbf{x}(\bar{p}-k)\left[L, \partial_{j}\right]\left(u_{j}\right) \\
-\int_{\Omega} \mathrm{d} \mathbf{x} \bar{u}_{i}\left(\left[L, \partial_{t}\right]\left(u_{i}\right)+\left[L, \partial_{i}\right](p)\right)-\int_{\Omega} \mathrm{d} \mathbf{x} \bar{u}_{i}\left[L, \partial_{j}\right]\left(u_{i} u_{j}\right) \\
+\frac{1}{R e} \int_{\Omega} \mathrm{d} \mathbf{x} \bar{u}_{i}\left[L, \partial_{j j}\right]\left(u_{i}\right)
\end{aligned}
$$

after some partial integrations. One observes the usual dissipation of kinetic energy $E$ due to the viscous terms, as well as the transport term involving the subgrid stress tensor: $\tau_{i j} \partial_{j} \bar{u}_{i}$. Moreover, one notices a contribution arising from the fact that the filtered velocity field is no longer solenoidal involving $(\bar{p}-k)\left[L, \partial_{j}\right]\left(u_{j}\right)$. Finally, four terms emerge characterizing the effects of the commutator errors in the momentum equations. The magnitude of the various terms and commutator errors can be quantified by explicitly evaluating the different integrals during a simulation or by post-processing direct numerical simulation databases (see also [11]). 
The commutator brackets in (3) and (4) satisfy two fundamental algebraic identities. For any two filters $L_{1}$ and $L_{2}$ then

$$
\left[L_{1} L_{2}, S\right]=\left[L_{1}, S\right] L_{2}+L_{1}\left[L_{2}, S\right]
$$

which is known as Germano's identity [2]. Likewise,

$$
\left[L_{1},\left[L_{2}, S\right]\right]+\left[L_{2},\left[S, L_{1}\right]\right]+\left[S,\left[L_{1}, L_{2}\right]\right]=0
$$

which is recognized as Jacobi's identity. These identities are also satisfied if $S$ is replaced by $\partial_{t}$ or $\partial_{j}$. This shows that the structure of the large-eddy closure problem has some formal similarities to the Poisson-bracket in classical mechanics. In that context, Germano's identity is known as the Leibniz rule. The identities (8) and (9) can be used to guide (dynamic) subgrid modelling of the central commutator brackets. The dynamic procedure has been successfully applied to the modelling of the turbulent stress tensor [19] and can also be extended to improve base models developed for the commutator errors. An alternative modelling approach, based on regularization principles [9] will be discussed in section 4. However, before considering explicit subgrid modelling for the commutator errors, we turn to estimating the magnitude of the commutator errors relative to the turbulent stress fluxes, in the next section.

\section{Magnitude of commutator errors and turbulent stress fluxes}

This section introduces general high order filters and shows that the commutator errors and turbulent stress fluxes associated with these filters are formally of the same order in the filterwidth $\Delta$. Subsequently, limitations on the non-uniformities of the filter are characterized, and situations are identified in which commutator errors are much smaller than the turbulent stress fluxes. In what follows, we consider only filters that do not depend explicitly on time, i.e., $\left[L, \partial_{t}\right]=0$.

In principle, all commutator errors in (3) and (4) require explicit parametrization in much the same way as the turbulent stress fluxes do. However, in practice one would like to address this subgrid closure only for those contributions that are actually dynamically relevant. The contributions of the commutator errors have been considered unimportant by some authors, provided a suitable class of so-called high order filters [20] would be adopted. Different, symmetric filters were constructed such as in $[14,15]$ in which it was shown that the commutator errors could be made of arbitrarily high order in $\Delta$ by raising the order of the filter appropriately.

Although it is correct that the commutator errors can be made small with the proper filter, we will complement this statement by showing that with that same filter the turbulent stress fluxes are also reduced at the same rate. More specifically, in the general case both contributions scale with the same power of the filter-width $\Delta$ [21]. Earlier studies will be extended by allowing the filter to be skewed [11], which may occur, e.g., near solid boundaries, or for filters expressed directly in terms of an underlying non-uniform computational grid, i.e., for filters whose support is set equal to an integer number of grid-cells. Hence, we find that the size of the commutator errors may not be controlled independently of the magnitude of the turbulent stress fluxes simply by adopting appropriate, high order, non-uniform filters.

The importance of the commutator errors relative to the turbulent stress fluxes may be established by taking the following steps. We first introduce general $N$ th order filters by requiring that $L\left(x^{k}\right)=x^{k}$ for $k=0,1, \ldots, N-1[21,20]$. Without significant loss of generality, we may consider compact support filters in one spatial dimension. If we introduce the integration-variable $y=(\xi-x) / \Delta(x)$ in (1) we may write

$$
\bar{u}(x, t)=\int_{\mathcal{I}_{x}} \mathrm{~d} y H(x, x+\Delta(x) y) u(x+\Delta(x) y, t)
$$


where the support of the filter is given by

$$
\mathcal{I}_{x}=\left\{y \in \mathbb{R} \mid \frac{\sigma(x)-1}{2} \leqslant y \leqslant \frac{\sigma(x)+1}{2}\right\}, \quad \sigma(x)=\frac{\Delta_{+}-\Delta_{-}}{\Delta_{+}+\Delta_{-}}
$$

in terms of the 'normalized skewness' of the filter, $\sigma$, which satisfies $|\sigma| \leqslant 1$. The filtering of $x^{k}$ may now be expressed as

$$
\begin{aligned}
\overline{x^{k}} & =\int_{\mathcal{I}_{x}} \mathrm{~d} y H(x, x+\Delta(x) y)(x+\Delta(x) y)^{k} \\
& =\sum_{m=0}^{k}\left(\begin{array}{l}
k \\
m
\end{array}\right) x^{k-m} \Delta^{m}(x) m ! M_{m}(x),
\end{aligned}
$$

in terms of the 'moments'

$$
M_{m}(x)=\frac{1}{m !} \int_{\mathcal{I}_{x}} \mathrm{~d} y H(x, x+\Delta(x) y) y^{m} .
$$

By requiring the characteristic filter function $H$ to be such that $M_{m}(x)=\delta_{m 0}$ for $m=$ $0,1, \ldots, N-1$ an $N$ th order filter is obtained [21, 22].

Application of an $N$ th order filter to a smooth signal $u$ yields:

$$
\bar{u}-u=\sum_{m=N}^{\infty}\left(\Delta^{m}(x) M_{m}(x)\right) u^{(m)}=\Delta^{N}(x) M_{N}(x) u^{(N)}+\cdots
$$

where $u^{(m)}$ denotes the $m$ th spatial derivative of $u$. We observe that the effect of the filter, expressed as the difference between $u$ and $\bar{u}$, scales with the $N$ th power of the filter-width $\Delta$. In addition, the moment $M_{N}$ determines the magnitude of the filter's effect. For symmetric filters all odd-numbered moments are 0 ; hence such filters have even order. Important examples are the top-hat and Gaussian filters which are of second order. By perturbing such a filter to become skewed, e.g., at nonzero $\sigma$ or if the form of $H$ is altered appropriately, the formal order will typically reduce to 1 , although higher order skewed filters may readily be determined as well [21]. We next investigate the effect of these filters on the commutator error and turbulent stress flux.

To quantify the various subgrid contributions in more detail we consider the following decomposition of a typical nonlinearity:

$$
\begin{aligned}
\overline{\partial_{x}\left(u^{2}\right)} & =\partial_{x}\left(\bar{u}^{2}\right)+\partial_{x}\left(\overline{u^{2}}-\bar{u}^{2}\right)+\left\{\overline{\partial_{x}\left(u^{2}\right)}-\partial_{x}\left(\overline{u^{2}}\right)\right\} \\
& =\partial_{x}\left(\bar{u}^{2}\right)+\partial_{x}([L, S](u))+\left[L, \partial_{x}\right](S(u))
\end{aligned}
$$

in which we distinguish a mean flux contribution $\partial_{x}\left(\bar{u}^{2}\right)$ next to the turbulent stress flux $\partial_{x}([L, S](u))$ and the commutator error $\left[L, \partial_{x}\right](S(u))$. Analogous to (14) we may find expressions for $\overline{\partial_{x}\left(u^{2}\right)}$ and $\overline{u^{2}}$ and hence also for $\partial_{x}\left(\overline{u^{2}}\right)$. Based on this, after some calculation the commutator error $\left[L, \partial_{x}\right](S(u))$ can be written as

$$
\begin{aligned}
{\left[L, \partial_{x}\right]\left(u^{2}\right) } & =-\sum_{m=N}^{\infty}\left(\Delta^{m} M_{m}\right)^{\prime}\left(\left(u^{2}\right)^{(m)}\right) \\
& =-\sum_{m=N}^{\infty}\left(m \Delta^{m-1} \Delta^{\prime} M_{m}+\Delta^{m} M_{m}^{\prime}\right)\left(\left(u^{2}\right)^{(m)}\right)
\end{aligned}
$$

where the prime indicates differentiation with respect to $x$. Combination of $\overline{u^{2}}$ and $\bar{u}^{2}$ allows the turbulent stress tensor to be expressed as 


$$
\begin{aligned}
& {[L, S](u)=\sum_{m=N}^{\infty}\left(\Delta^{m} M_{m}\right) g_{m}(x)} \\
& g_{m}(x)=\left[\left(u^{2}\right)^{(m)}-2 u u^{(m)}\right]-u^{(m)}(x) \sum_{k=N}^{\infty}\left(\Delta^{k} M_{k}\right) u^{(k)}(x)
\end{aligned}
$$

and, correspondingly, we find for the turbulent stress flux

$$
\partial_{x}([L, S](u))=\sum_{m=N}^{\infty}\left(\Delta^{m} M_{m}\right)^{\prime} g_{m}(x)+\left(\Delta^{m} M_{m}\right) g_{m}^{\prime}(x) .
$$

Expressions (16) and (19) form a basis for discussing the magnitude of the commutator error and turbulent stress flux for general $N$ th order filters.

To estimate the magnitude of the commutator error and the turbulent stress flux, we first collect some basic properties. We describe the non-uniform filter-width in (1) by $\Delta_{ \pm}(x)=\varepsilon f_{ \pm}(x)$ with constant $\varepsilon$ such that $0 \leqslant \varepsilon \ll 1$. We assume that $f_{ \pm}$are positive, bounded functions, with bounded derivatives.

- Correspondingly, $\Delta(x)=\varepsilon f(x)$ with $f=f_{+}+f_{-} \geqslant 0$, and $\Delta^{\prime}=\varepsilon f^{\prime}$. Since $f$ and $f^{\prime}$ are bounded, we observe that both $\Delta$ and $\Delta^{\prime}$ are of the same order in $\varepsilon$. In particular, $\Delta^{m}=\varepsilon^{m} f^{m}$ and $\Delta^{m-1} \Delta^{\prime}=\varepsilon^{m} f^{\prime} f^{m-1}$ are both of $m$-th order in the parameter $\varepsilon$.

- The normalized skewness $\sigma$ and its derivative $\sigma^{\prime}$ do not reduce to 0 as $\varepsilon \rightarrow 0$; these quantities are of zeroth order in $\varepsilon$.

- The moments $M_{m}$ may be evaluated in more detail when the characteristic filter function $H(x, \xi)$ has a convergent Taylor expansion at every $x$ in the domain: $H(x, \xi)=$ $\sum_{k=0} a_{k}(x)(\xi-x)^{k}$ with $a_{0} \neq 0$. In this case the dominant contribution to $M_{m}$ is of zeroth order in $\Delta$, i.e., $M_{m}$ does not reduce to 0 as $\varepsilon \rightarrow 0$. This case excludes 'singular' filters for which $\Delta^{m} M_{m}=$ const for some $m$.

- It may be shown that $M_{m}^{\prime}$ is governed by three different terms; (i) a contribution arising from $\partial_{x} H(x, \xi)+\partial_{\xi} H(x, \xi) \neq 0$, i.e., $H(x, \xi) \neq \mathcal{H}(x-\xi)$ expressing the fact that the characteristic filter function is not of convolution type, (ii) a contribution proportional to $\Delta^{\prime}$ and (iii) a contribution proportional to $\sigma^{\prime}$. The dominant contributions to $M_{m}^{\prime}$ may be shown to be of zeroth order in $\varepsilon$ in case $H$ has a convergent Taylor expansion.

- Finally, we consider $g_{m}$ as arise in (17). Since $\left(u^{2}\right)^{\prime}-2 u u^{\prime}=0$ it is convenient to distinguish the case $m=1$ from $m \geqslant 2$. The contribution at $m=1$ only arises as $N=1$ and we observe that typically the dominant contribution to $g_{1}$ is proportional to $\Delta M_{1}$. In case $m \geqslant 2$ the dominant term arises from $\left(u^{2}\right)^{(m)}-2 u u^{(m)}$ and is also of zeroth order in $\varepsilon$.

The actual magnitude of the various contributions in (16) and (19) depends strongly on the specific non-uniformity of the upper and lower bounding functions $\Delta_{ \pm}$and the specific filter that was adopted in a given application. However, the typical dominant scaling with $\varepsilon$ can be inferred quite generally.

After performing these basic estimates, we may summarize our findings as follows. Turning to (16) both contributions under the summation are of $m$-th order in $\varepsilon$; the first term because $M_{m}$ is of $O\left(\varepsilon^{0}\right)$ and $\Delta^{m-1} \Delta^{\prime} \sim O\left(\varepsilon^{m}\right)$, the second because $M_{m}^{\prime}$ is of $O\left(\varepsilon^{0}\right)$. Therefore, $\left[L, \partial_{x}\right]\left(u^{2}\right) \sim O\left(\varepsilon^{N}\right)$. Likewise, if the order of the filter $N \geqslant 2$, the turbulent stress tensor in (17) scales with $\varepsilon^{N}$ since $g_{m}$ is of $O\left(\varepsilon^{0}\right)$ for $m \geqslant 2$. If $N=1$ we observed $g_{1} \sim \Delta M_{1}$ and so $[L, S](u) \sim \varepsilon^{2}$. Consequently, the turbulent stress flux in (19) is of order $\varepsilon^{N}$ with a characteristic contribution $\sim \Delta^{\prime} \Delta^{N-1}$ as $N \geqslant 2$. In case $N=1$ the turbulent stress flux typically scales with $\varepsilon^{2}$ with a characteristic term $\sim \Delta^{\prime} \Delta$. Hence, the two subgrid 
contributions to the total flux in (15) are formally of equal order of magnitude if $N \geqslant 2$. If $N=1$ the situation becomes even more striking; the commutator error scales with terms of $O(\varepsilon)$ while the turbulent stress flux scales with terms which are formally of $O\left(\varepsilon^{2}\right)$. In this case, which corresponds, e.g., to the application of a skewed top-hat or Gaussian filter, the formal order of magnitude of the commutator error is even larger than that of the turbulent stress fluxes.

The detailed evaluation of the turbulent stress flux and the commutator error indicates an alternative route toward (some) independent control over the ratio of these contributions. It is well known that commutator errors are zero if and only if the filter is a strict convolution filter. Therefore, it will be intuitively clear that if the spatial filter is 'close' to this case, the dynamic implications of the commutator errors are likely to be small. Specifically, this implies that variations in $\Delta$ and in $\sigma$ as well as the deviation of $H(x, \xi)$ from a function $\mathcal{H}(x-\xi)$ should be kept sufficiently small. For general $N$ th order filters, a separate control over the commutator error can be obtained only by restraining these non-uniformities. In such cases one could argue that explicit modelling of the commutator errors may not be required. Conversely, for sufficiently large variations of these filter properties, the fluxes associated with the commutator errors may become significant and require explicit treatment. An a priori analysis along these lines has been described in [11], using direct numerical simulation data of a turbulent mixing flow. This numerical analysis illustrates the estimates above and indicates that for significant filter non-uniformities the commutator errors can no longer be neglected.

Further estimates of the dynamic significance of the commutator errors may be obtained via Fourier analysis. Roughly speaking, this approach relates the spatial non-uniformities of the filter to a typical length-scale of the flow-structure being considered. To illustrate. we focus attention on a symmetric top-hat filter; the analysis for general filters is beyond the scope of this paper and will be published elsewhere. In a single-mode analysis, one assumes a solution $u=\sin (k x)$ and finds explicitly

$\partial_{x}([L, S](u))+\left[L, \partial_{x}\right]\left(u^{2}\right)=C(k \Delta)\left\{k \sin (2 k x)+\frac{\Delta^{\prime}}{\Delta}(\cos (2 k x)-1)\right\}$

where the flux-amplitude function $C$ is given by

$$
C(z)=\frac{z \sin (z)-2+2 \cos (z)}{z^{2}}=-\frac{1}{12} z^{2}+\frac{1}{180} z^{4}+O\left(z^{6}\right)
$$

showing a dominant scaling with $(k \Delta)^{2}$ as $|k \Delta| \ll 1$. The two contributions to the flux in (20) have 'weights' $k$ and $\left(\Delta^{\prime} / \Delta\right)$, respectively, from which one infers that if variations in $\Delta$ are sufficiently 'mild', i.e. $\left|\Delta^{\prime}\right| \ll|k \Delta|$ then filter-width non-uniformity can be disregarded. In general, for symmetric second order filters one may show that

$$
\frac{\left\|\left[L, \partial_{x}\right](S(u))\right\|}{\left\|\partial_{x}([L, S](u))\right\|} \sim \frac{\left|\Delta^{\prime} / \Delta\right|}{|k|}
$$

where $\|\cdot\|$ denotes the $L_{2}$-norm. Again $\left|\Delta^{\prime}\right| \ll|k \Delta|$ is required for the dynamics of a structure with wave number $k$ not to be significantly altered by filter-width irregularities.

As sketched in the introduction, the accumulated effect of the commutator error arises as a parcel of fluid moves through regions of decreasing/increasing filter width. In the Lagrangian framework, the material time-derivative of the filter-width is hence a precise measure for quantifying the commutator error effects in physical space [11, 12]. Consequently, control over $D \Delta / D t=\partial_{t} \Delta+u_{j} \partial_{j} \Delta$ can be helpful in appropriately restricting filter-width variations along flow-paths. Keeping the commutator error effects small enough is particularly difficult in turbulent boundary layers. In a priori estimates using direct numerical simulation of a temporal boundary layer flow [6] it appeared that close to solid walls the flux contribution 
from the commutator errors is about half as large as the turbulent stress fluxes, and explicit modelling of commutator error appears to be necessary. Various possible closures for the commutator error may be proposed [23]. The next section considers the commutator errors in similarity modelling and in the Leray regularization approach.

\section{Similarity and regularization modelling of commutator errors}

This section considers situations in which the spatial variations in the filter-width are sufficiently localized to motivate explicit modelling of the commutator errors. We consider only similarity modelling and Leray regularization. Specifically, we will extend Bardina's approach [13] to include commutator errors and derive the implied subgrid models for the turbulent stresses and the commutator error, arising from non-uniform Leray regularization $[8,9]$. Subsequently, we compare these two large-eddy models in a simple but illustrative situation by considering 'ramp-cliff' solutions of the viscous Burgers equation running across a region of strong filter non-uniformity.

Bardina's similarity model for the turbulent stress tensor arises by applying the definition of $\tau_{i j}=[L, S]\left(u_{i}, u_{j}\right)$ to the filtered solution $\bar{u}_{i}$, i.e.,

$$
\tau_{i j} \rightarrow m_{i j}^{B}=[L, S]\left(\bar{u}_{i}, \bar{u}_{j}\right)=\overline{\bar{u}}_{i} \bar{u}_{j}-\overline{\bar{u}}_{i} \overline{\bar{u}}_{j}
$$

Extending this idea to the commutator error suggests the following parametrization:

$$
\left[L, \partial_{j}\right]\left(u_{i} u_{j}\right) \rightarrow\left[L, \partial_{j}\right]\left(\bar{u}_{i} \bar{u}_{j}\right) .
$$

In a turbulent boundary layer flow the model contributions (23) and (24), were shown to provide a high correlation with the actual turbulent stress tensor and commutator errors respectively [6]. The extended Bardina model may be motivated in an alternative manner. Instead of distinguishing separate closure problems for the commutator error and the turbulent stress fluxes, we may consider the full non-uniformly filtered convective flux, i.e., $\overline{\partial_{j}\left(u_{i} u_{j}\right)}-\partial_{j}\left(\bar{u}_{i} \bar{u}_{j}\right)[24]$. One may verify that

$$
\begin{aligned}
\overline{\partial_{j}\left(u_{i} u_{j}\right)} & =\partial_{j}\left(\bar{u}_{i} \bar{u}_{j}\right)+\left\{\overline{\partial_{j}\left(u_{i} u_{j}\right)}-\partial_{j}\left(\bar{u}_{i} \bar{u}_{j}\right)\right\} \\
& =\partial_{j}\left(\bar{u}_{i} \bar{u}_{j}\right)+\left[L, \partial_{j} \circ S\right]\left(u_{i}, u_{j}\right) .
\end{aligned}
$$

The similarity closure arises as before, i.e., $\left[L, \partial_{j} \circ S\right]\left(u_{i}, u_{j}\right) \rightarrow\left[L, \partial_{j} \circ S\right]\left(\bar{u}_{i}, \bar{u}_{j}\right)$, where:

$$
\left[L, \partial_{j} \circ S\right]\left(\bar{u}_{i}, \bar{u}_{j}\right)=\left[L, \partial_{j}\right] \circ S\left(\bar{u}_{i}, \bar{u}_{j}\right)+\partial_{j} \circ[L, S]\left(\bar{u}_{i}, \bar{u}_{j}\right)
$$

In rewriting this model, use was made of the Leibniz rule (8), applied to the operators $L, \partial_{j}$ and $S$. The similarity modelling of the separate closure problems for the commutator error, cf (24), and the turbulent stress fluxes, cf (23), is hence re-obtained directly from the similarity modelling of the full convective-flux. By adopting the same modelling assumptions for both the turbulent stress tensor and the commutator error the combined model can be implemented at reduced computational cost.

Recently, the Leray regularization principle [8] was revisited in the context of large-eddy simulation [9]. In this approach the convective fluxes $u_{j} \partial_{j} u_{i}$ are replaced by $\bar{u}_{j} \partial_{j} u_{i}$, i.e., the solution $\mathbf{u}$ is convected with a smoothed velocity $\overline{\mathbf{u}}$. The governing Leray equations for incompressible flow are given by

$$
\partial_{j} u_{j}=0, \quad \partial_{t} u_{i}+\bar{u}_{j} \partial_{j} u_{i}+\partial_{i} p-\frac{1}{R e} \partial_{j j} u_{i}=0 .
$$

This formulation can be written in terms of $\left\{\bar{u}_{i}, \bar{p}\right\}$ by assuming the existence of a (formal) inverse $L^{-1}$ of $L$, i.e., $u_{j}=L^{-1}\left(\bar{u}_{j}\right)$. After some calculation, one obtains the filtered 
momentum equation as

$$
\begin{aligned}
\partial_{t} \bar{u}_{i}+\partial_{j}\left(\bar{u}_{i} \bar{u}_{j}\right) & +\partial_{i} \bar{p}-\frac{1}{R e} \partial_{j j} \bar{u}_{i}=-\left(\left[L, \partial_{t}\right]\left(u_{i}\right)+\left\{\partial_{j}\left(m_{i j}^{L}\right)+\overline{u_{i} \partial_{j} \bar{u}_{j}}\right\}+\left[L, \partial_{j}\right]\left(S\left(u_{i}, \bar{u}_{j}\right)\right)\right. \\
+ & {\left.\left[L, \partial_{i}\right](p)-\frac{1}{R e}\left[L, \partial_{j j}\right]\left(u_{i}\right)\right) . }
\end{aligned}
$$

The divergence of the turbulent stress tensor $\partial_{j} \tau_{i j}$ in (4) is represented in terms of the Leray model $m_{i j}^{L}=\overline{\bar{u}}_{j} u_{i}-\bar{u}_{j} \bar{u}_{i}$ and an additional term associated with the divergence of the filtered velocity field:

$$
\partial_{j} \tau_{i j} \rightarrow \partial_{j}\left(m_{i j}^{L}\right)+\overline{u_{i} \partial_{j} \bar{u}_{j}}
$$

in which the commutator error is expressed as $\left[L, \partial_{j}\right]\left(u_{i} u_{j}\right) \rightarrow\left[L, \partial_{j}\right]\left(\bar{u}_{j} u_{i}\right)$. The other commutator errors are identical to those in (4) with the understanding that in actual simulations every occurrence of an unfiltered flow-variable implies the application of $L^{-1}$ to the smoothed field. The Leray model is known to provide good predictions of three-dimensional turbulent mixing at arbitrarily high Reynolds number when a uniform filter is used [9].

To illustrate the dynamics associated with commutator errors and the quality of their explicit modelling we consider simulations of the one-dimensional viscous Burgers equation. While the dynamics of the viscous Burgers equation is clearly different from that of the Navier-Stokes equations, both models for fluid flow are quadratically nonlinear and exhibit the same commutator errors, except for the pressure term. First, we describe the numerical method, then some a priori analysis is given to establish the magnitude of the commutator errors for specific non-uniformities in filter width and finally we illustrate the performance of the explicit similarity and regularization models for the commutator errors.

We consider the one-dimensional viscous Burgers equation

$$
\partial_{t} u+\partial_{x}\left(\frac{1}{2} u^{2}\right)-\frac{1}{R e} \partial_{x x} u=0
$$

and adopt $R e=500$. The numerical treatment of this equation follows the standard method of lines [1]. Introducing $u_{j}(t)=u\left(x_{j}, t\right)$ as the discrete solution at location $x_{j}$ at time $t$, the method of lines implies the semi-discretization

$$
\frac{\mathrm{d} u_{j}}{\mathrm{~d} t}+f_{j}=0, \quad u_{j}(0)=u_{0}\left(x_{j}\right)
$$

where $f_{j}$ denotes the numerical flux at $x_{j}$ and $u_{0}$ the initial condition. We adopt periodic boundary conditions and obtain the numerical flux by second order accurate finite differencing on a non-uniform grid. The approximation of the convective term $(1 / 2) \partial_{x}\left(u^{2}\right)$ follows from

$$
\partial_{x}\left(u^{2}\right) \rightarrow \delta_{x}\left(u^{2}\right)_{j}=\frac{u_{j+1}^{2}-u_{j-1}^{2}}{x_{j+1}-x_{j-1}}
$$

and the approximation of the viscous term $\partial_{x x} u$ results from

$$
\partial_{x x} u \rightarrow \delta_{x x}(u)_{j}=a_{j, j+1} u_{j+1}-a_{j, j} u_{j}+a_{j, j-1} u_{j-1}
$$

where

$$
\begin{aligned}
& a_{j, j+1}=\frac{2}{\left(x_{j+1}-x_{j}\right)\left(x_{j+1}-x_{j-1}\right)} \\
& a_{j, j}=\frac{2}{\left(x_{j+1}-x_{j}\right)\left(x_{j}-x_{j-1}\right)} \\
& a_{j, j-1}=\frac{2}{\left(x_{j}-x_{j-1}\right)\left(x_{j+1}-x_{j-1}\right)} .
\end{aligned}
$$




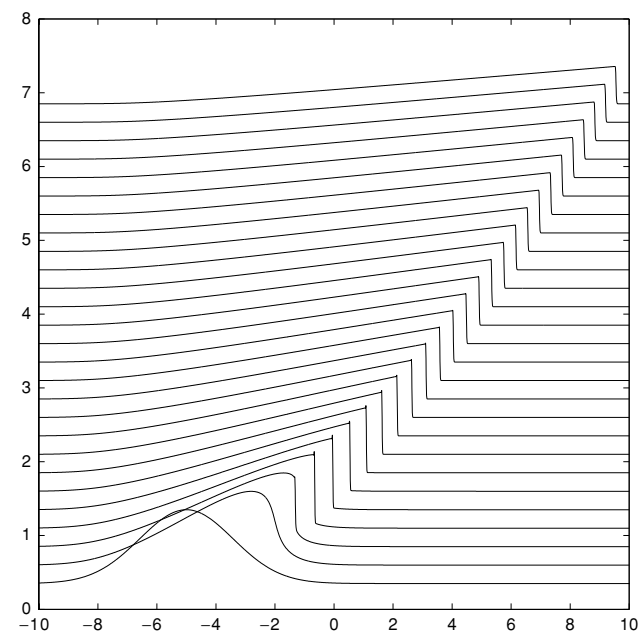

Figure 1. Solution to the Burgers equation initiated with a Gaussian profile, developing into a ramp-cliff structure at $R e=500$. The solution is shown at times $t=0,1,2, \ldots$.

On a uniform grid with grid-spacing $h$ these weights reduce to $a_{j, j-1}=a_{j, j+1}=1 / h^{2}$ and $a_{j, j}=2 / h^{2}$ which is recognized as the second-order accurate finite difference scheme for the second-order derivative.

The system of ordinary differential equations in (31) is integrated in time using explicit time-integration, restricted by stability time-steps. In fact, we use time-steps associated with local stability that follow from the Courant-Friedrich-Lewy condition (CFL). This implies

$$
\Delta t=\min _{j}\left(\Delta t_{j}\right), \quad \Delta t_{j}=\Gamma \frac{\Delta x_{j}}{\left|u_{j}\right|}, \quad \Delta x_{j}=\frac{x_{j+1}-x_{j-1}}{2}
$$

where the CFL-number $\Gamma$ is chosen consistent with the stability requirements of the adopted time-integration method. Use was made of either the explicit Euler forward method at a low value $\Gamma=0.1$ or the compact storage, four-stage explicit Runge-Kutta method with $\Gamma=1.5[1]$.

The implementation of the Bardina model follows the standard LES-template (4), applied to the one-dimensional Burgers equation. For the Leray model we used (27) as the basis for the implementation. Since the numerical illustration is in one spatial dimension only, computational resources do not represent a limiting factor. Consequently, we can avoid effects of numerical errors [25-27] by adopting sufficiently high spatial resolution. Typically, we show results in which the Burgers equation is discretized using grids with $N=2048$ intervals. This is more than adequate for obtaining the nearly grid-independent, unsteady solution, as was verified independently by comparing results on different grids. An example of a developing ramp-cliff solution is shown in figure 1 . The initial velocity profile consists of a small positive value to which a Gaussian profile is added. In the examples shown, this Gaussian profile is centred around $x=-5$ and its width was selected as 5 .

The explicit filtering required in the Bardina and Leray models, or when filtering direct numerical simulation data, was implemented using the trapezoidal rule for evaluating the top-hat filter. Specifically, we define

$$
\bar{u}_{j}(t)=\frac{1}{x_{j+n_{1}}-x_{j-n_{2}}} \int_{x_{j-n_{2}}}^{x_{j+n_{1}}} u(\xi, t) \mathrm{d} \xi
$$



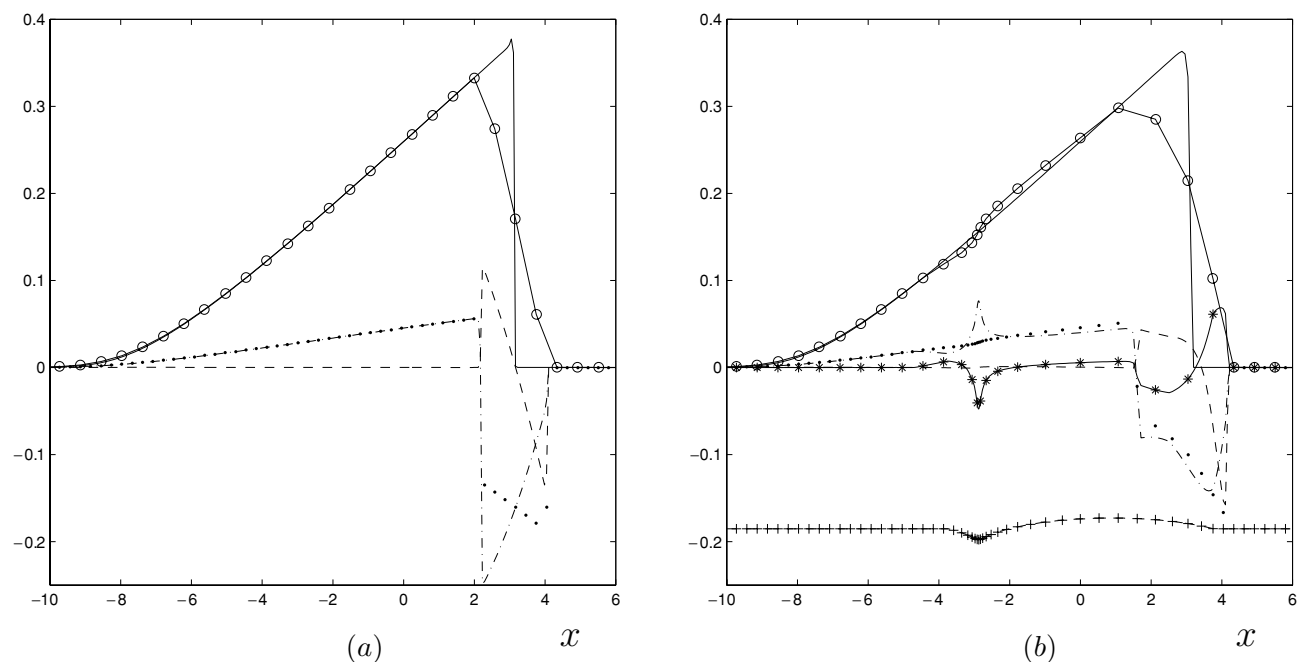

Figure 2. Snapshot of the solution (multiplied by 1/2) (solid) and filtered solution (solid; markers $o$ ) obtained at $N=2048$. Convective flux: total (dots), mean (dash-dotted), turbulent stress (dashed), commutator error (solid with $*$ ). In $(a)$ we use $\Delta=\ell / 16$, i.e., computational filter-width $n_{1}=n_{2}=64$ and in $(b)$ the non-uniform case with $A=1 / 2$ and the same values of $n_{1}$ and $n_{2}$ is shown. Underneath in $(b)$, the grid-spacing (minus 0.2 ) as a function of $x$ is presented.

which covers $n_{1}+n_{2}$ grid-cells. In terms of the grid, a non-uniform filter-width $\Delta_{j}=$ $x_{j+n_{1}}-x_{j-n_{2}}$ is obtained together with a normalized skewness

$$
\sigma_{j}=\frac{x_{j+n_{1}}-2 x_{j}+x_{j-n_{2}}}{x_{j+n_{1}}-x_{j-n_{2}}} .
$$

The integration over $\xi$ in (36) is evaluated using the composite trapezoidal rule. This rule is sufficiently accurate, because in one dimension we can allow for large numbers of intervals, i.e., take $n_{1}$ and $n_{2}$ large enough to imply negligible discretization errors. This setting allows one to approximate the grid-independent large-eddy solution corresponding to a fixed filterwidth distribution. In fact, if the resolution $N$ is increased by a factor $k$ then $n_{1}$ and $n_{2}$ need to be increased with the same factor in order to properly represent the filter-width non-uniformity as a fixed, externally specified aspect of the flow problem. If, instead, only $N$ were increased at fixed $n_{1}, n_{2}$, this would lead to a decrease of the filter-width with increasing resolution. This situation corresponds to a gradual convergence to a direct numerical simulation and not to the desired large-eddy formulation consistent with a given filter-width distribution.

We consider a non-uniform grid with grid-spacing $h_{i}=(\ell / N)\left(1+g_{i}\right)$ where $\ell$ is the length of the domain, which was set equal to 20 in our simulations. The grid is non-uniform around $i=N / 2$ with

$$
g_{i}=A \sin \left(2 \pi \frac{(i-N / 2)}{(N(m-2 q) / m)}\right), \quad \frac{q N}{m} \leqslant i \leqslant \frac{(m-q) N}{m}
$$

and 0 otherwise. We use $q=3, m=8$ and $N=2^{n}$ with $n$ sufficiently large. The parameter $A<1$ controls the ratio between largest and smallest intervals $(1+A) /(1-A)$.

In figure 2 we collected the contributions to the total convective flux for a representative uniform and non-uniform case. The solution and the filtered solution both display the 'rampcliff' structure. The total flux in figure $2(a)$ is piecewise linear and the turbulent stress flux is localized in the cliff-region. In figure $2(b)$ the filter-width non-uniformity strongly influences 


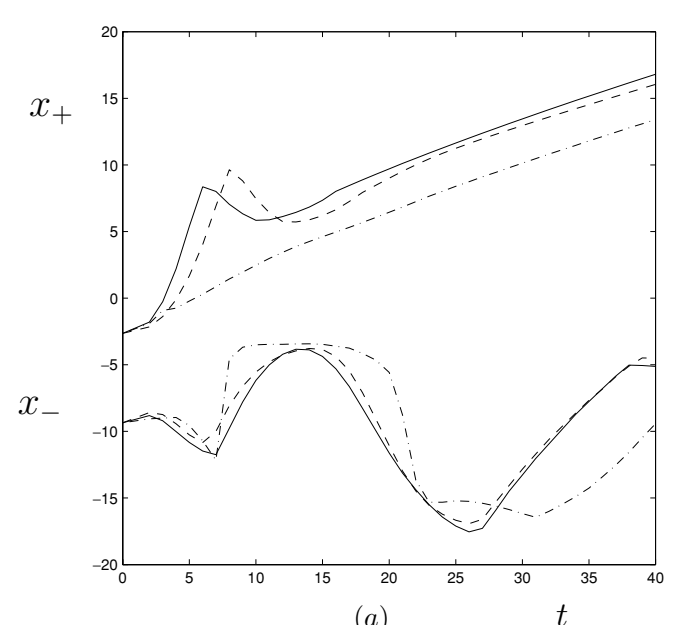

(a)

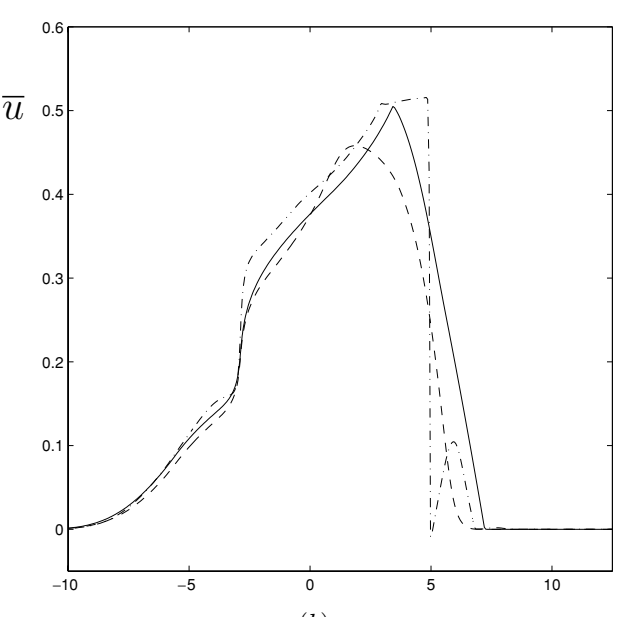

(b) $\quad x$

Figure 3. Location of the head $x_{+}$of the cliff (upper curves) and the tail $x_{-}$of the ramp (lower curves) in ( $a$ ) and in (b) snapshot of the filtered solution: filtered Burgers (solid), Leray (dashed) and Bardina (dash-dotted) for $A=0.85$ and $n_{1}=n_{2}=64$ at $N=2048$.

the mean flux on the 'ramp side' near $x=-3$. The commutator error compensates for this such that the total flux remains nearly linear in $x$. The commutator error and the turbulent stress flux have comparable magnitude in the filtered cliff-region $2 \lesssim x \lesssim 4$, in which these contributions are seen to partially counteract each other.

In figure 3(a) we show the locations of the front and back of the ramp-cliff solution as a function of time. These locations are defined to be where $|u|$ equals $\max (|u|) / 20$. Upon comparing filtered Burgers results with predictions from the Leray and Bardina parametrizations, one finds the Leray results are more accurate. The $L_{2}$-norm of the fluxes show that the commutator error is about $1 / 3-1 / 2$ the value of the turbulent stress flux in this case. The Leray model also preserves better the qualitative properties of the filtered Burgers solution cf figure 3(b). The Bardina parametrization creates additional variations in the solution, which do not correspond to the physics of the filtered Burgers equation in this rather extreme non-uniform case.

\section{Concluding remarks}

In this paper the commutator errors associated with non-uniform filtering in large-eddy simulation were studied. For a general class of non-uniform filter operators the filtered, incompressible Navier-Stokes equations were derived and all closure terms were identified. Besides the turbulent stress contributions, commutator errors were shown to arise and a Lagrangian interpretation of their dynamical effects was provided. The order of magnitude of the commutator errors and the turbulent stress fluxes was derived for arbitrary high order filters, with bounded moments. The main result of this analysis is that both closure contributions scale with the same order of the filter-width, when the spatial non-uniformity is nonzero. This implies that while an increase in the order of the spatial filter allows control over the magnitude of the commutator error, the flux due to the turbulent stress is affected simultaneously in the same order of magnitude. Hence, an independent control over the commutator errors cannot be obtained through the application of a general high order filter. 
A more detailed analysis of the commutator errors and turbulent stress fluxes shows that the commutator errors may be reduced in size by explicitly restricting the variations in the filter-width $\Delta^{\prime}$ and normalized skewness $\sigma^{\prime}$ of the filter. This suggests employing only gradually varying filter-widths in complex geometries, from the point of view of avoiding explicitly modelling of the commutator errors. In view of maintaining appropriate efficiency in large-eddy simulations of turbulent flows in/around complex geometries it may be required to allow for sharp variations in $\Delta$ and $\sigma$. In such cases the dynamic importance of the commutator errors summons an explicit parametrization of the commutator errors.

For symmetric filters, so long as $\left|\Delta^{\prime}\right| \ll|k \Delta|$, where $1 / k$ is indicative of the length-scale of the flow-feature, explicit modelling is not required. However, at sufficiently large grid non-uniformities explicit modelling will become necessary. An extension of the similarity approach was formulated and compared with the Leray regularization approach. The Leray parametrization captures both the flux due to the turbulent stresses and the commutator errors in one model. Consequently, it combines computational efficiency with high accuracy. This result motivates the use of the Leray model in complex flows and it stimulates the study of more general regularization approaches for the closure of commutator errors in large-eddy simulation. As a first illustration, the prediction of the solution to the non-uniformly filtered Burgers equation was studied and the Leray approach was found to provide higher accuracy than the full similarity modelling. The extension to turbulent flow in three dimensions is presently being considered and will be presented elsewhere.

\section{Acknowledgments}

BJG would like to acknowledge support from the Los Alamos Turbulence Working Group and Center for Nonlinear Studies. DDH was partially funded by the Department of Energy under contracts W-7405-ENG-36 and the DOE Office of Sciences Advanced Scientific Computing Research (ASCR) Applied Mathematical Research program.

\section{References}

[1] Geurts B J 2003 Elements of Direct and Large-Eddy Simulation (Flourtown, PA: R T Edwards Inc.)

[2] Germano M 1992 J. Fluid Mech. 238325

[3] Friedrich R and Arnal M 1990 J. Wind Eng. Ind. Aerodyn. 35101

[4] Le H and Moin P 1994 Report No. TF-58 Department of Mechanical Engineering (Stanford University)

[5] Ghosal S and Moin P 1995 J. Comput. Phys. 286229

[6] Geurts B J, Vreman A W and Kuerten J G M 1994 Proc. 74th AGARD Symp. vol 551 p 51

[7] Ghosal S 1999 AIAA J. 37425

[8] Leray J 1934 Acta Math. 63193

[9] Geurts B J and Holm D D 2003 Phys. Fluids 15 L13

[10] McComb W D 1990 The Physics of Fluid Turbulence (Oxford: Oxford University Press)

[11] van der Bos F and Geurts B J 2005 Phys. Fluids 17035108

[12] van der Bos F and Geurts B J 2005 Phys. Fluids 17075101

[13] Bardina J, Ferziger J H and Reynolds W C 1984 Report TF-19 Department of Mechanical Engineering (Stanford University)

[14] H van der Ven 1995 Phys. Fluids 71171

[15] Vasilyev O V, Lund T S and Moin P 1998 J. Comput. Phys. 14682

[16] Goldstein H, Poole C P and Safko J L 2002 Classical Mechanics 3rd edn (Reading, MA: Addison Wesley)

[17] Kuerten J G M, Geurts B J, Vreman A W and Germano M 1999 Phys. Fluids 113778

[18] Pope S B 2000 Turbulent Flows (Cambridge: Cambridge University Press)

[19] Germano M, Piomelli U, Moin P and Cabot W H 1991 Phys. Fluids 31760

[20] Daubechies I 1990 IEEE Trans. Inf. Theory 36961 
[21] Geurts B J, Vreman A W, Kuerten J G M and van Buuren R 1997 Proc. Direct and Large-Eddy Simulation II (Grenoble) ed P R Voke, L Kleiser and J P Chollet (Dordrecht: Kluwer) p 47

[22] Geurts B J 1997 Phys. Fluids 93585

[23] Geurts B J 2002 Advances in LES of Complex Flows: Proc. ed R Friedrich and W Rodi (Dordrecht: Kluwer) p 13

[24] Vreman A W and Geurts B J 2002 Advances in Turbulence IV ed I P Castro, P E Hancock and T G Thomas (Barcelona: CIMNE) p 199

[25] Geurts B J and Fröhlich J 2002 Phys. Fluids 14 L41

[26] Meyers J, Geurts B J and Baelmans M 2003 Phys. Fluids 152740

[27] Meyers J, Geurts B J and Baelmans M 2005 Phys. Fluids 17045108 